\title{
Prenatal stress impairs recognition memory and leads to neurodevelopmental deficits in the hippocampus of adolescent rats with early acute pentylenetetrazole-kindling
}

\author{
Kübra Çelik $^{1} \oplus$, Petek Bilim ${ }^{1,2} \oplus$, Gurur Garip $^{3} \oplus$, Burak Durmaz $^{4}$, Eser Yıldırım Sözmen ${ }^{4}$, Meral \\ Baka $^{3} \odot$
}

${ }^{1}$ Department of Neuroscience, Ege University Institute of Health Sciences, Izmir, Turkey

${ }^{2}$ Department of Psychology, Toros University Faculty of Economics, Business Administration and Social Sciences, Mersin, Turkey

${ }^{3}$ Department of Histology and Embryology, Ege University School of Medicine, Izmir, Turkey

${ }^{4}$ Department of Medical Biochemistry, Ege University School of Medicine, İmir, Turkey

\section{ABSTRACT}

Objectives: This study aimed to investigate the effects of prenatal stress (PS) on hippocampus of early acute pentylenetetrazole (PTZ)-kindled offspring in adolescence. Recognition memory, morphological changes and synaptophysin levels in hippocampus were evaluated.

Methods: Restraint stress was induced to a group of pregnant dams and non-stressed (NA) group remained undisturbed. Next, male and female offspring were divided as 1. PS-PTZ, 2. PS -control, 3. NA-PTZ and 4. NA-control ( $\mathrm{n}=12$ in each group). The object recognition test was performed following PTZ injection (45 $\mathrm{mg} / \mathrm{kg}$ ) on postnatal day 10 (P10). Brains were collected on postnatal day 35 (P35) to determine neuronal density and synaptophysin expression by immuno/-histological studies. Further, oxidative stress products in hippocampus were analyzed with different biochemical assays.

Results: PS impaired recognition memory in PTZ group significantly $(p=0.03)$; however, the impairment of PS was reversible in control group compared to PTZ $(p=0.04)$. Furthermore, PS caused neuronal loss in CA1 $(p=0.01)$ and decreased synaptophysin expression in the CA3 area of hippocampus in PTZ group $(p=0.03)$. PS also increased the oxidative stress markers in PTZ group significantly $(p<0.05)$.

Conclusions: These results suggest that PS causes neurodevelopmental deficits in adolescent hippocampus and recognition memory after early-life seizures prominently. However, the damage of only PS in adolescence can be reversible. Therefore, the effects of PS in the adult hippocampus and other regions of brain need to be further studied

Keywords: prenatal stress, hippocampus, PTZ-kindling, seizure, recognition memory

$\mathrm{T}$ raumas in embryonic development usually cause major impacts in the neonatal period and later life of the infant [1]. Prenatal stress (PS) especially increases the tendency and frequency of seizures causing neurodevelopmental deficits in the brain [2]. PS impairs the regulation of the hypothalamic-pituitaryadrenal axis and decreases the seizure threshold through glucocorticoid enhancement [3]. Furthermore, suppression of the inhibitor GABAergic neurons and constant depolarization of glutamatergic neurons [4, neurodevelopmental deficits in the hippocampus of adolescent rats with early acute pentylenetetrazole-kindling. Eur Res J 2021;7(4):340-347. DOI: 10.18621/eurj.801699

Address for correspondence: Kübra Çelik, Ege University Institute of Health Sciences, Department of Neuroscience, Kazımdirik Mah., 35100 Bornova, İzmir, Turkey.E-mail: kubracelik94@gmail.com 
5] impairs the neonatal hippocampus through volume loss, dendritic atrophy, suppression of synaptic transmission $[6,7,8]$. Since the hippocampus is crucial for learning and memory processing, the damage of PS may cause cognitive deficits such as recognition problems in later life. However, recognition memory requires multiple pathways such as visual, entorhinal and perirhinal cortex. The role of hippocampus in recognition process is not known well. In this case, several studies indicated that PS increases seizure activity or improves learning and memory retention associated with hippocampus $[9,10]$. Nevertheless, that improvement or impairment depends on timing, duration, intensity of PS and gender [1]. High levels of glucocorticoids as a physiological response to PS stimulate postsynaptic excitatory receptors by over-releasing glutamate. Consequently, increased intracellular $\mathrm{Ca}+2$ concentration leads to seizure susceptibility and oxidative stress which may cause neuronal loss in the developing hippocampus $[11,12]$. However, the long-term effects of PS and early acute seizure on the oxidative stress markers, synaptic transmission and neuronal denstiy of hippocampus need to be clarified. Therefore, we investigated the effects of PS on hippocampus of early acute pentylenetetrazole (PTZ) kindled offspring in adolescence. We aimed to understand the cognitive, biochemical and histological outcomes when hippocampus development is disrupted by acute early life seizures following PS exposure.

\section{METHODS}

\section{Animals}

Adult female Wistar albino rats (200-400 g) were obtained from Ege University, Laboratory of Animal Research and Application Center. They were housed with ad libitum food and water under 20-25Co, 12L/12D laboratory conditions. The estrous cycle of female rats was determined by vaginal smear and they were housed with male rats overnight. Next day, vagi- nal plugs were observed and presumed as the first day of pregnancy. Pregnant rats were divided into PS and non-stress (NA) groups. After the birth, offspring ( $\mathrm{n}=$ 12) in each group stayed in their home cages until weaning and separated by their genders. Experimental groups were conducted as 1. PS-PTZ, 2. PS-control, 3. NA-PTZ and 4. NA-control. This study was carried out after the approval of the local ethical committee (2017-082). The experimental design is shown in Fig. 1.

\section{Prenatal Stress Procedure}

Restraint stress was induced to a group of female rats $(n=6)$ between day 12.5-17 of their gestations individually due to hippocampus begins to form on embryonic day 12 in rats [14]. They were restrained in a Plexiglass transparent cylinder $(19 \mathrm{~cm} \times 6 \mathrm{~cm})$ three times/day for 45 min under two bulbs (100W) as described previously [15]. NA group of pregnant rats was not disturbed.

\section{Pentylentetrazole Injection}

Equal amount of male and female offspring from different litters received diluted PTZ $(45 \mathrm{mg} / \mathrm{kg})$ in saline intraperitoneally on postnatal day 10 (Sigma Aldrich-P6500) [16]. After the injection, they were transferred to a transparent cage to observe behavioral outcomes. 4 Tonic-clonic generalized seizures characterized by myoclonic jerks, chewing scraping, contractions in all limbs, gradually decreasing tonic movements and freezing were observed in all animals for at least 1 hour following PTZ injection.

\section{Object Recognition Test}

Object recognition test was developed by Ennaceur and Delacour states that the animal learns the object in natural conditions and retains information in memory depending on discovery behavior without any penalty or reward [17]. Object recognition test was performed to 10 animals in each group. First day, the animals were placed into a $60 \times 60 \times 40$ box for $5 \mathrm{~min}$

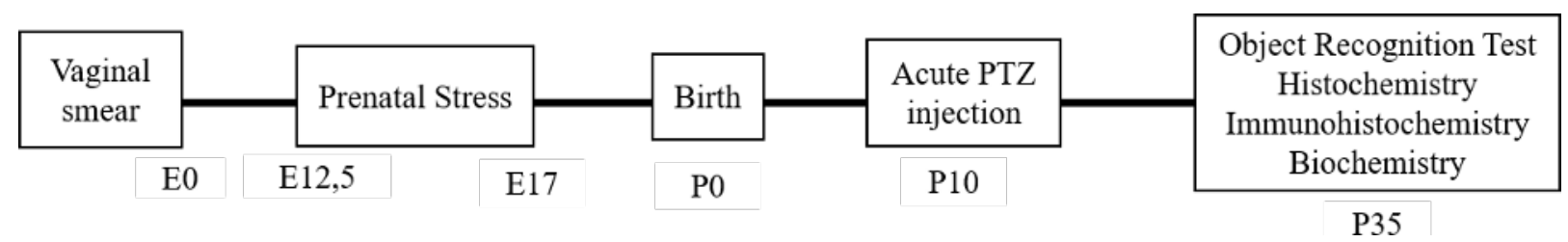

Fig. 1. The experimental design. $\mathbf{E}=$ embryonic day, $\mathbf{P}=$ postnatal day. 
and expected to explore the box. Second day, two familiar objects were placed on different quadrants of the box symmetrically and rats discovered the objects for 10 minutes. Third day, the familiar object was replaced with a different shaped, sized and colored object. The animals placed into the box to explore familiar and novel objects for 10 minutes. All of the trials were recorded and recognition time was measured by two different stopwatches and mean was considered. The box and objects were cleaned with $70 \%$ alcohol between trials to avoid odor factor. Discrimination time was defined as when the animal approached the object less than $2 \mathrm{~cm}$, sniffed, pawed and self-cleaned alongside the object. The discrimination index was calculated as follows: (Discrimination index $=($ new object $/$ total exploration $) \times 100$. $)$

\section{Immuno/-histochemistry and Image Analysis}

The animals $(n=6)$ in each group were anesthetized and intra-cardiac perfusion was administered with $4 \%$ formaldehyde in $0.1 \mathrm{M}$ phosphate-buffered saline (PBS) on P35. Brains were removed and washed in $0.1 \mathrm{M}$ cacodylate buffer after storing in $4 \%$ formaldehyde in $0.1 \mathrm{M}$ PBS for 3 days, respectively. Next day, brain slices were embedded into paraffin and sectioned $(5 \mu \mathrm{m})$ for stainings. Firstly, Haematoxylin $\&$ Eosin staining (Merck, Germany) was 5 used to examine morphological differences of hippocampal regions. Secondly, another group of sections was incubated with anti-synaptophysin (1:100, Sigma Aldrich) primary antibody to detect synaptophysin, a presynaptic protein localized in synaptic vesicles [13]. Next day, the sections were washed and incubated with anti-mouse IgG (1:200, Sigma Aldrich) for 40 min. Finally, after DAB staining synaptophysin expressions were examined by light microscopy and microphotographs were captured (Olympus BX-51, light microscope, Olympus C-5050 digital camera). Synaptophysin immunoreactivity and neuronal density were examined in CA1 and CA3 areas by Image $J$ (https://imagej.nih.gov/ij/). For this assessment, hippocampus of each animal was randomly chosen for unbiased counting and the average of 10 images from different sections was obtained by two researchers to ensure objectivity [18].

\section{Biochemistry}

Another group of brains $(n=5)$ was removed without intra-cardiac perfusion and hippocampus was dissected from each brain $(n=5)$. Further, tissues were homogenized in phosphate buffer $(0.5 \mathrm{M}, \mathrm{pH}: 7.0)$, $(1 / 10: \mathrm{w} / \mathrm{v})$ and homogenates were centrifuged for 5 min at $700 \times \mathrm{g}$ at $4{ }^{\circ} \mathrm{C}$. Supernatants were collected immediately to determine myeloperoxidase (MPO), catalase (CAT) and thiobarbituric acid reactive substances (TBARS) levels. Tissue homogenates were incubated with TBARS solution (0.12 M TBA in $15 \%$ TCA and $1 \% \mathrm{HCl}$ ) for $30 \mathrm{~min}$ at $95 \mathrm{Co}$. TBARS levels were calculated using 1,1,3,3, tetramethoxypropane standard curve [19]. Hydrogen peroxide degradation was recorded at $240 \mathrm{~nm}$ by spectrophotometry and catalase activity was calculated [20]. MPO activities were measured following these stages: Homogenates were centrifuged at $10.000 \mathrm{rpm}$ for $15 \mathrm{~min}$. Pellets were re-homogenized in $0.5 \%$ HETAB (hexadecyltrimethylammonium bromide) in PBS (50 mM, pH: 6.0 ) and centrifuged $10.000 \mathrm{rpm}$ for $10 \mathrm{~min}$. Supernatants were added to a reactive solution containing $0.5 \mathrm{M}$ o-dianisidine. Hydrogen peroxide solution (20 $\mathrm{mM}$ ) was added and 6 absorbance was recorded at 492 $\mathrm{nm}$ with a microplate reader for $3 \mathrm{~min}$ with 15 -sec intervals. Finally, MPO activity was calculated using the standard curve [21].

\section{Statistical Analysis}

IBM SPSS Statistics 22.0 was used for statistical analysis. One-way ANOVA and post hoc Bonferonni tests were performed in normal distributed data according to Shapiro-Wilk test. Mann-Whitney test was used to compare PTZ and hypoxia subgroups in PS and NA groups for non-parametric data. The significance was considered as $p<0.05$ and data is shown as mean \pm standard error of the mean (S.E.M.)

\section{RESULTS}

\section{Object Recognition Test}

The discrimination index revealed that NA-PTZ group discriminated the new object better than PSPTZ group ( $p=0.03)$. However, the control offspring of the PS group $(76.88 \pm 9.95)$ discriminated the new object better than PS-PTZ group ( $p=0.04,42.91 \pm$ 8.87) significantly. The object discrimination was worse in PTZ group compared to control for both PS and NA groups (Fig. 2). There was no statistical sig- 


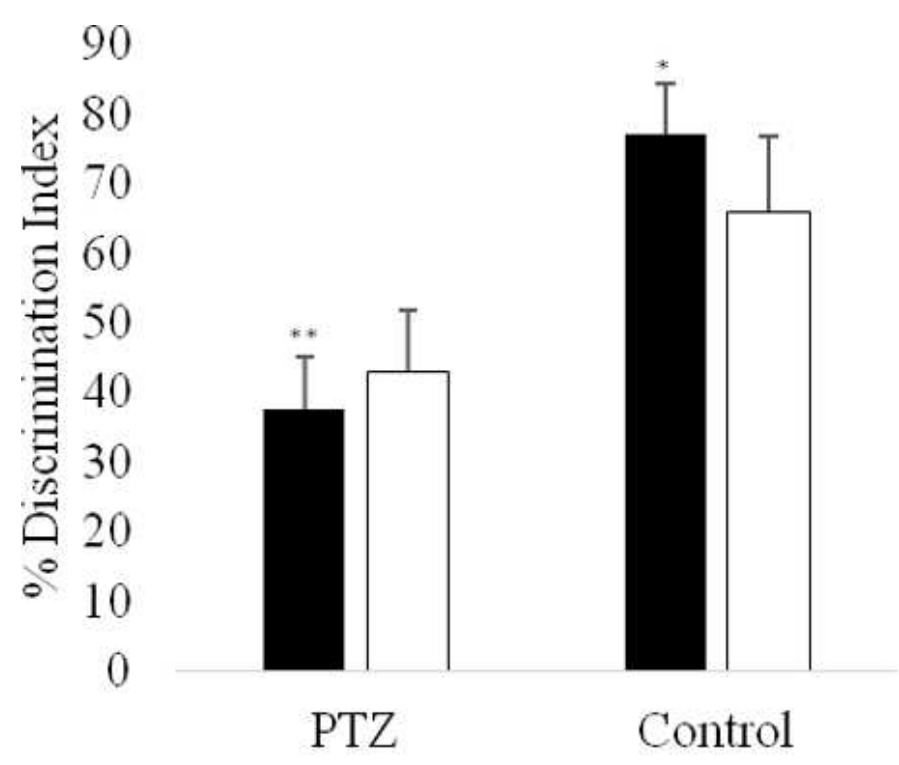

\section{- Prenatal Stress $\square$ Non-Stress}

Fig. 2. Discrimination index of object recognition test. PS decreased the object discrimination in PTZ group and increased in control significantly ( $n=10$ in each group). * $p=0.04$ vs PSPTZ, $* * \mathbf{p}=0.03$ vs NA-control

nificance between PS and NA in the control group.

\section{Haematoxylin \& Eosin (H\&E)}

CA1 and CA3 areas of hippocampus were examined to compare morphological differences (Fig. 3a and Fig. 3b). Gliosis and characteristic oligodendroglial nuclei were quite evident in PS group. Neuron loss was especially prominent in the CA1 area of PSPTZ group significantly $(p=0.015)$ (Table 1$)$. Further, large cavities between glias were observed and 7 gliosis in alveus proved the developmental deficits, especially in CA1. There was not a statistical difference between groups in CA3.

\section{Synaptophysin Expressions}

Synaptophysin immunostaining was performed on CA1 and CA3 in each group and indicated as mean percentage. Post hoc test revealed that PS reduced synaptophysin immunoreactivity in CA3 of PTZ group ( $p=0.03,38.60 \pm 0.86)$, contrary it increased synaptophysin in control group significantly $(p=0.02$, $38.11 \pm 1.14)$. There was no significant difference between groups in CA1 even though the mean percentage of PS-control was higher than NA-control (Fig. 4a and Fig. 4b).

\section{TBARS, MPO and CAT Levels}

The current data demonstrated that PS increased TBARS $(p=0.04)$ and MPO levels $(p=0.01)$ in PTZ group significantly. Although the means of NA groups were higher than PS, there was not a statistical difference between CAT levels (Table 2). Furthermore, there was no statistical difference between PTZ and control groups in NA offspring. The results of biochemical assays proved that lipid peroxidation and oxidative stress were higher in PS-PTZ group significantly.
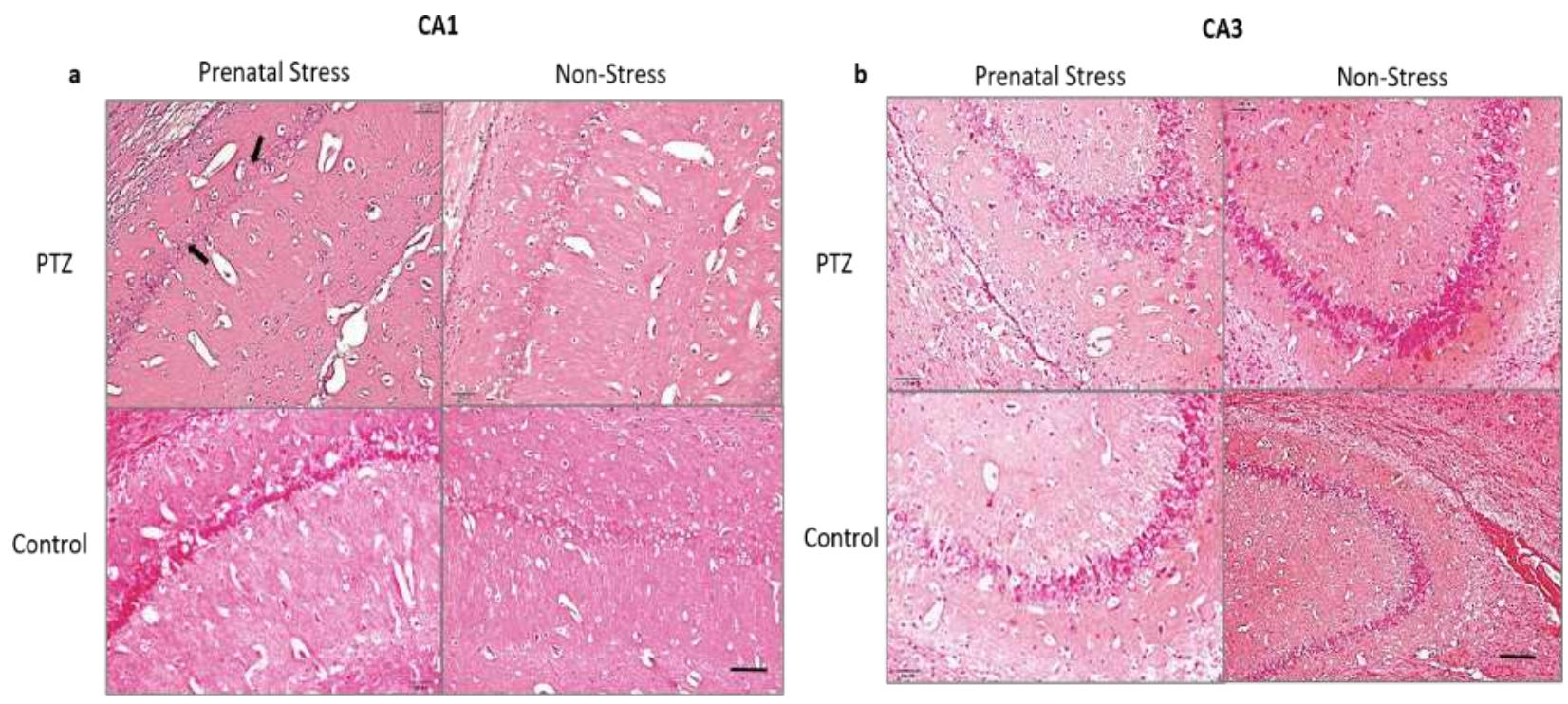

Fig. 3. Pyramidal layers of CA1 and CA3 areas H\&E staining shows the morphological differences in the pyramidal layers in CA1 (a) and CA3 (b) regions. Neuron loss in PTZ group of PS was seen by arrows in CA1 (scale bar=50 $\mu \mathrm{m}$, the magnification is $20 \times$ ) 
Table 1. Quantitive measurement of the neuronal density by H\&E staining

\begin{tabular}{lcc}
\hline Groups $(\mathbf{n}=\mathbf{5})$ & CA1 & CA3 \\
\hline Prenatal Stress (PS) & & \\
PTZ & $129.83 \pm 21.818^{*}$ & $188.17 \pm 26.077$ \\
Control & $281.17 \pm 25.375$ & $223.17 \pm 21.917$ \\
Non-Stressed (NA) & & \\
PTZ & $235.33 \pm 45.238$ & $329.50 \pm 33.985$ \\
Control & $285.83 \pm 25.088$ & $683.50 \pm 60.668$ \\
\hline
\end{tabular}

Data is presented as mean \pm standard error of the mean (S.E.M.). CA1 and CA3 areas detected in hippocampus of each animal under light microscopy (20× magnification) and these areas were randomly chosen. The average of 10 images from different sections was obtained and counted by two researchers using Image J (https://imagej.nih.gov/ij/). PTZ = pentylenetetrazole, ${ }^{*} p$ $=0.015$ vs NA-control

a

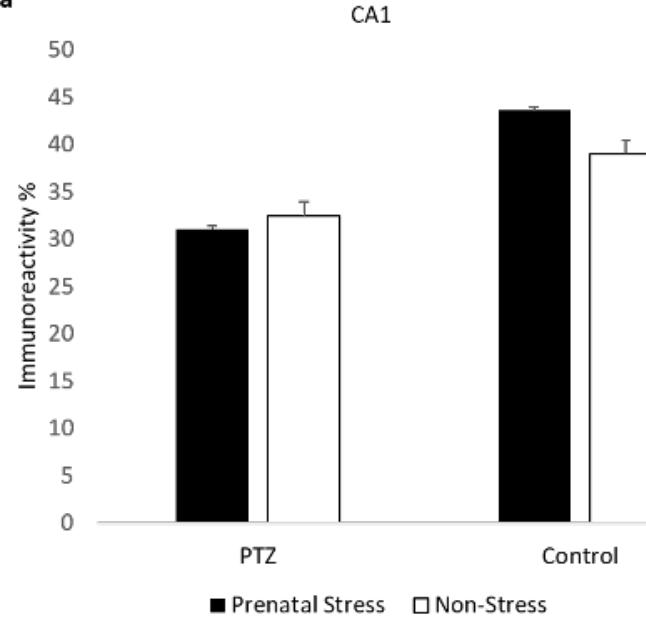

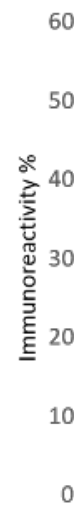

60

50

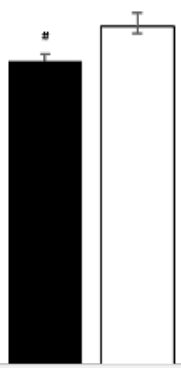

PTZ

- Prenatal Stress $\square$ Non-Stress
$\mathrm{CA} 3$

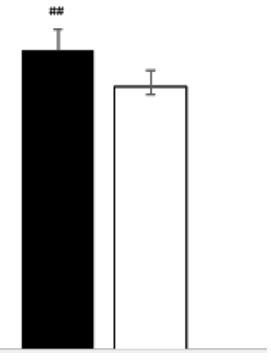

Control

Fig. 4. Synaptophysin immunoreactivity. Synaptophysin positive areas were measured on $20 \times$ microphotographs by two researchers using Image $J$ and the result is showed as a mean percentage. PS decreased the immunoreactivity in the $C A 3$ region of the PTZ group and increased in control offspring significantly. There was no statistical significance between groups in the CA1 region. $* p=0.03$ vs NA-PTZ, $* * p=0.02$ vs PS-PTZ

Table 2. TBARS, MPO and CAT levels.

\begin{tabular}{lccc}
\hline Groups $(\mathbf{n}=\mathbf{6})$ & $\begin{array}{c}\text { TBARS } \\
\text { (nmol/mgprt) }\end{array}$ & $\begin{array}{c}\text { CAT } \\
\text { (U/mgprt) }\end{array}$ & $\begin{array}{c}\text { MPO } \\
\text { (U/mgprt) }\end{array}$ \\
\hline Prenatal Stress (PS) & & & $0.50 \pm 0.009^{* *}$ \\
PTZ & $3.99 \pm 1.08^{*}$ & $0.44 \pm 0.15$ & $0.06 \pm 0.01$ \\
Control & $2.69 \pm 0.62$ & $0.38 \pm 0.15$ & \\
Non-Stressed (NA) & & & $0.13 \pm 0.04$ \\
PTZ & $2.13 \pm 0.25$ & $0.45 \pm 0.06$ & $0.05 \pm 0.02$ \\
Control & $2.79 \pm 0.21$ & $0.24 \pm 0.10$ & \\
\hline
\end{tabular}

Data is shown as mean \pm standard error of the mean (S.E.M.). In PS groups, values of TBARS, CAT, and MPO in PTZ group were higher than in control group. TBARS $=$ thiobarbituric acid reactive substances, $\mathrm{CAT}=$ catalase, $\mathrm{MPO}=$ myeloperoxidase, PTZ $=$ pentylenetetrazole. ${ }^{*} p=0.04$ vs NA-PTZ, ${ }^{*} p=0.01$ vs NA-PTZ 


\section{DISCUSSION}

PS is a risk factor for the occurrence and progression of seizures [22]. Therefore, it may lead to many neurodevelopmental and cognitive deficits in the developing brain. In this study, it was questioned whether PS causes neurodevelopmental deficits and recognition impairment in hippocampus of adolescent offspring with acute PTZ-kindling in early life. In this context, CA1 and CA3 regions of the hippocampus were examined regarding the detection of synaptic transmission and morphological changes. TBARS, MPO and CAT assays were conducted to demonstrate lipid peroxidation and antioxidant levels of the whole hippocampus. Furthermore, the object recognition test was performed to investigate recognition abilities and whether the hippocampus involves in the recognition process.

PS has different impacts on the hippocampus which is crucial for memory processing. However, the effects of PS on recognition memory of adolescent rats with early-life seizures are not known well. Ahmadi et al. [23] demonstrated that PTZ-kindled rats have exploratory deficits on object recognition. Current results similarly showed that recognition memory of PTZ-kindled rats was impaired associated with the neuron loss and reduced synaptophysin levels in CA1. Indeed, H\&E staining showed that CA1 was more vulnerable than CA3 to the effects of PS. In contrary, PS increased the object discrimination in control group compared to PTZ-kindled group regarding synaptophysin levels in CA3. PS caused neuronal and synaptic damage in PTZ group, however that damage was reversible in the control group. These results referred that the long-term effects of PS enhance or reduce cognitive capabilities depending on seizure activity in the adolescent brain $[9,10]$. Another important finding was that hippocampus is essential for the object recognition process. However, it is not the primary area for memory storage and retention since the entorhinal, perirhinal, and visual cortex are involved in possible mechanisms [24-27]. Therefore, despite the deficits in hippocampus PS increased the discrimination index in control group. Further, these regions of the cortex must be investigated to understand effects of PS on recognition memory.

TBARS, CAT, and MPO levels were determined to investigate lipid peroxidation and oxidative stress.
Several studies revealed that stress in the prenatal period increases TBARS, which is an end-product of lipid peroxidation [28-30]. Besides that, seizure activity also increases lipid peroxidation $[31,32]$. Similarly, this study demonstrated that PS increases TBARS and MPO levels in PTZ group significantly. However there was no significance between NA groups. These results referred to epileptic seizures are associated with increased oxidative damage [33] due to reperfusion injury as well as the polymorphonuclear leukocyte recruitment in the brain $[34,35]$. Consequently, increased oxidative damage and insignificant levels of catalase indicated that PS causes developmental disruptions by changing the oxidant $\&$ antioxidant balance of brain [36].

\section{CONCLUSION}

Consequently, PS disrupted recognition memory, neuronal density and synaptic transmission in hippocampus of PTZ group in adolescence. Further studies are needed to understand the effects of PS in adult brain and other possible mechanisms as well as gender differences. This study provided a multidisciplinary evidence for the effects of PS on the recognition memory and hippocampus of adolescent rats with early acute PTZ-kindling.

\section{Authors' Contribution}

Study Conception: KÇ, PB, MB; Study Design: KÇ, PB, MB; Supervision: EYS, MB; Funding: Ege University Scientific Research Project Cordination 18SBE-004; Materials: Ege University Scientific Research Project Cordination 18-SBE-004; Data Collection and/or Processing: KÇ, PB, GG, BD, EYS; Statistical Analysis and/or Data Interpretation: KÇ, PB, GG, BD, EYS; Literature Review: KÇ, PB, GG, BD, EYS; Manuscript Preparation: KÇ and Critical Review: EYS, MB.

\section{Conflict of interest}

The authors disclosed no conflict of interest during the preparation or publication of this manuscript.

\section{Funding}

This work was supported by the Scientific Research Project Coordination of Ege University, İzmir, 
TURKEY (18-SBE-004/ 2018).

\section{Acknowledgement}

We would like to thank Assoc. Prof. Dr. Timur Köse for his support on statistical analysis and The Department of Histology and Embryology staff for their assistance in the laboratory work.

\section{REFERENCES}

1. Weinstock M. The long-term behavioural consequences of prenatal stress. Neurosci Biobehav Rev 2008;32:1073-86.

2. Qulu L, Daniels WM, Mabandla M. Exposure to prenatal stress enhances the development of seizures in young rats. Metab Brain Dis 2012;27:399-404.

3. Lopim GM, Gutierre RC, da Silva, EA, Arida RM. Physical exercise during pregnancy minimizes PTZ-induced behavioral manifestations in prenatally stressed offspring. Dev Psychobiol 2020;62:240-49.

4. Krugers HJ, Karst H, Joels M. Interactions between noradrenaline and corticosteroids in the brain: from electrical activity to cognitive performance. Front Cell Neurosci 2012;6:15.

5. Weinstock M. The potential influence of maternal stress hormones on development and mental health of the offspring. Brain Behav Immun 2005;19:296-308.

6. Jia N, Yang K, Sun Q, Cai Q, Li H, Cheng D, et al. Prenatal stress causes dendritic atrophy of pyramidal neurons in hippocampal CA3 region by glutamate in offspring rats. Dev Neurobiol 2010;70:114-25.

7. Lai MC, Huang LT. Effects of early life stress on neuroendocrine and neurobehavior: mechanisms and implications. Pediatr Neonatol 2011;52:122-9.

8. Schoenfeld TJ, McCausland HC, Morris HD, Padmanaban V, Cameron HA. Stress and loss of adult neurogenesis differentially reduce hippocampal volume. Biol Psychiatry 2017;82:914-23.

9. Fujioka T, Fujioka A, Tan N, Chowdhury GMI, Mouri H, Sakata Y, et.al. Mild prenatal stress enhances learning performance in the non-adopted rat offspring. Neuroscience 2001;103:301-7.

10. Cannizzaro C, Plescia F, Martire M, Gagliano M, Cannizzaro G, Mantia G, et. al. Single, intense prenatal stress decreases emotionality and enhances learning performance in the adolescent rat offspring: Interaction with a brief, daily maternal separation. Behav. Brain Res 2006;169:128-36.

11. Zhu Z, Li X, Chen W, Zhao Y, Li H, Qing C, et. al. Prenatal stress causes gender-dependent neuronal loss and oxidative stress in rat hippocampus. J. Neurosci Res 2004;78:837-44.

12. Ikonomidou C, Kaindl AM. Neuronal death and oxidative stress in the developing brain. Antioxidants Redox Signal 2011;14:1535-50.

13. Tannenberg RK, Dodd PR. Cell Damage/excitotoxicity. excitotoxicity and neurodegenerative disease, in: Encyclopedia of Basic Epilepsy Research. Elsevier, 2009: pp.114-9.

14. Andersen P, Morris R, Amaral D, O’Keefe J, Bliss T, The hip- pocampus book. Oxford University Press 2007.

15. Edwards H, Dortok D, Tam J, Won D, Burnham WM. Prenatal stress alters seizure thresholds and the development of kindled seizures in infant and adult rats. Horm Behav 2002;42:437-47. 16. Nassiri-Asl M, Shariati-Rad S, Zamansoltani F. Anticonvulsive effects of intracerebroventricular administration of rutin in rats. Prog Neuropsychopharmacol Biol Psychiatry 2008;32:98993.

17. Ennaceur A, Delacour J. A new one-trial test for neurobiological studies of memory in rats. 1: Behavioral data. Behav. Brain Res 1988;31:47-59.

18. Turgut M, Uyanıkgil Y, Ateş U, Baka M, Yurtseven ME. Pinealectomy stimulates and exogenous melatonin inhibits harmful effects of epileptiform activity during pregnancy in the hippocampus of newborn rats: an immunohistochemical study. Childs Nerv Syst 2006;22:481-8.

19. Sözmen YE, Sözmen B, Girgin FK, Delen Y, Azarsiz E, Erdener D, et.al. Antioxidant enzymes and paraoxonase show a coactivity in preserving low-density lipoprotein from oxidation. Clin Exp Med 2001;1:195-9.

20. Aebi H. Catalase in vitro. Methods Enzymol 1984;105:1216.

21. Grisham MB, Anzueto Hernandez L, Granger DN. Xanthine oxidase and neutrophil infiltration in intestinal ischemia. Am J Physiol 1986;251(4 Pt 1):G567-74.

22. Sawyer NT, Escayg A. Stress and epilepsy: multiple models, multiple outcomes. J Clin Neurophysiol 2010;27:445-52.

23. Ahmadi M, Dufour JP, Seifritz E, Mirnajafi-Zadeh J, Saab BJ. The PTZ kindling mouse model of epilepsy exhibits exploratory drive deficits and aberrant activity amongst VTA dopamine neurons in both familiar and novel space. Behav Brain Res 2017;330:1-7.

24. Sanchez R, Dai W, Levada R, Lippman JJ, Jensen FE. AMPA/kainate receptor-mediated downregulation of GABAergic synaptic transmission by calcineurin after seizures in the developing rat brain. J Neurosci 2005;25:3442-51.

25. Barker GRI, Warburton EC. When is the hippocampus involved in recognition memory? J Neurosci 2011;31:10721-31.

26. Broadbent NJ, Gaskin S, Squire LR, Clark RE. Object recognition memory and the rodent hippocampus. Learn Mem 2010;17:5-11.

27. Detour J, Schroeder H, Desor D, Nehlig A. 5-month period of epilepsy impairs spatial memory, decreases anxiety, but spares object recognition in the lithium-pilocarpine model in adult rats. Epilepsia 2005;46:499-508.

28. Qulu L, Daniels WMU, Russell V, Mabandla, MV. Searsia chirindensis reverses the potentiating effect of prenatal stress on the development of febrile seizures and decreased plasma interleukin-1 $\beta$ levels. Neurosci Res 2016;103:54-8.

29. Watanabe H, Abe H, Takeuchi S, Tanaka R, Protective effect of microglial conditioning medium on neuronal damage induced by glutamate. Neurosci Lett 2000;289:53-6.

30. Yavin E, Glozman S, Green P. Docosahexaenoic acid accumulation in the prenatal brain prooxidant and antioxidant features. J Mol Neurosci 2001;16:229-35.

31. Lushchak VI, Bagnyukova TV. Hypoxia induces oxidative stress in tissues of a goby, the rotan Perccottus glenii. Comp 
Biochem Physiol B Biochem Mol Biol 2007;148:390-7. 32. Oliveira CC, de Oliveira CV, de Grigoletto J, Ribeiro LR, Funck VR, Grauncke ACB. Et al. Anticonvulsant activity of $\beta$ caryophyllene against pentylenetetrazol-induced seizures. Epilepsy Behav 2016;56:26-31.

33. Patel M. Mitochondrial dysfunction and oxidative stress: cause and consequence of epileptic seizures. Free Radic Biol Med 2004;37:1951-62.

34. Abraham M, Biju CR, Babu G. Neuroprotective effect of lacosamide and pregabalin on pentylenetetrazole induced seizure models in rat. Br Biomed Bull 2014;2:1-5.

35. Dhir A, Naidu PS, Kulkarni SK. Neuroprotective effect of nimesulide, a preferential COX-2 inhibitor, against pentylenetetrazol (PTZ)-induced chemical kindling and associated biochemical parameters in mice. Seizure 2007;16:691-7.

36. Bernhardt LK, Madhyastha S, Bairy L, Kishore A. Status of the brain antioxidant system at different growing periods after prenatal stress and N-Acetyl cysteine administration. Folia Neuropathol 2017;55:38-48. 